\title{
A review of microwave devices based on CVD-grown graphene with experimental demonstration
}

\author{
Wei-Bing Lu* , Hui Chen, and Zhen-Guo Liu \\ State Key Lab of Millimeter Waves, School of Information Science and Engineering, Southeast University, Nanjing 210096, \\ PR China
}

Received: 30 September 2018 / Accepted: 2 January 2019

\begin{abstract}
As a two-dimension planar material with zero-gap structure, graphene has a lot of outstanding properties in microwave frequency band, and the chemical vapor deposition (CVD) method can produce the large-scale graphene sheets with high quality for applications. Thus, the study about the microwave devices based on CVD-grown graphene has been aroused wide interests in the past few years. In this paper, mainly concentrating on the research by Chinese scientific groups, we review the development of microwave devices based on the CVD-grown graphene which are all validated by experiments, including attenuators, absorbers, antennas, electromagnetic interference (EMI) shielding and beam reconfiguration.
\end{abstract}

Keywords: CVD-grown grapheme / microwave devices / experimental demonstration

\section{Introduction}

Graphene, formed by carbon atoms in hexagonal ring sharp to structure honeycomb lattice, is the two-dimensional material with a thickness of $0.34 \mathrm{~nm}$. The planar hexagon dot array structure of graphene is exhibited in Figure 1a. The carbon atoms are in an $s p^{2}$ hybridization state, and each carbon atom is covalently bonded to three others and contribute an electron in $P$ orbit to form the $\pi$ bond [1-4]. The energy spectrum and energy band diagram of graphene are presented in Figure 1b, the valence band and the conduction band insect at one point (the Dirac point), which is similar to two tapered structures jointed and no gap between them, thus the graphene is also called zero-gap semiconductor [3].

Generally, the graphene can be synthesized through four methods. The first method is mechanical exfoliation of highly ordered pyrolytic graphite (HOPG) which is developed by Geim et al. of the University of Manchester in 2004 [5]. The graphene is firstly peeled from HOPG by the tape, and then transferred to $\mathrm{SiO}_{2}$ substrate. The second method is the epitaxial growth of graphene on silicon carbide ( $\mathrm{SiC}$ ). During the process, graphene layers are formed by heating $\mathrm{SiC}$ in high temperature over $1000^{\circ} \mathrm{C}[6]$. In the process of the third method which is the reduction of graphene oxide, the graphene oxide is reduced by reductant to produce uniform films containing monolayer or multilayer graphene $[7,8]$. The last one is the chemical vapor deposition (CVD) method. The graphene can be split from the carbonaceous gas on

\footnotetext{
* Corresponding author: wblu@seu.edu.cn
}

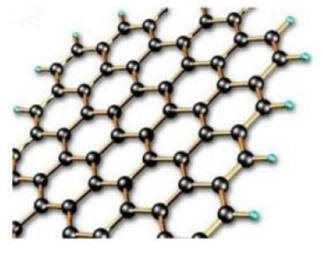

(a)

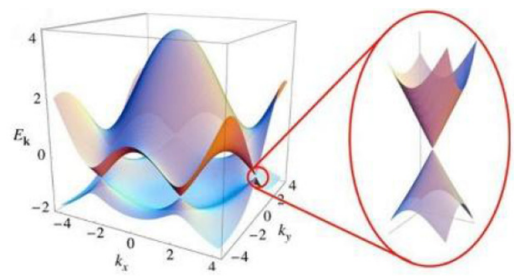

(b)
Fig. 1. (a) The planar hexagon dot array structure of graphene. (b) The energy spectrum and energy band diagram of graphene.

the surface of metal with catalytic function, which is the most widely used and most promising method to produce graphene considering compatibility in traditional semiconductor production lines and the needs of large-scale production [9]. A comparison of these methods is given in Table $1[10,11]$.

Due to its outstanding mechanical, optical, and electrical properties, the graphene has been widely used for microwave application in recent years [12]. Firstly, as the naturally thinnest material with the light transmittance of $97 \%-98 \%$, graphene paves the way to make the light and transparent devices [13]. Besides, one of the noticeable characteristic of graphene at microwave frequencies is the possibility to electronically modify its surface impedance, just like the tunable resistance, which provide graphene with opportunities to the application of tunable microwave components [14]. In addition, the graphene also has many other good properties, such as high thermal conductivity [15], high 
Table 1. The four methods of graphene synthesis.

\begin{tabular}{lll}
\hline Method & Quality & Area \\
\hline Mechanical exfoliation of highly ordered pyrolytic graphite (HOPG) & Very high & Small \\
Epitaxial growth of graphene on silicon carbide (SiC) & Medium & Large (3-4 inches wafers) \\
Reduction of graphene oxide (rGO) & Medium & Large \\
Chemical vapor deposition (CVD) & High & Very large (30 inches) \\
\hline
\end{tabular}

Table 2. Performance comparison of previously reported attenuators based on CVD-grown graphene.

\begin{tabular}{|c|c|c|c|c|c|}
\hline References & $\begin{array}{l}\left|S_{21}\right| \\
(\mathrm{dB})\end{array}$ & $\left|S_{11}\right|(\mathrm{dB})$ & $\begin{array}{l}\text { Frequency } \\
\text { band }(\mathrm{GHz})\end{array}$ & Structure & The function of GSSs in attenuator \\
\hline Ref. [18] & $2-15$ & $<-15 \mathrm{~dB}$ & $7-14.5$ & Two GSSs + SIW & Dissipate the EM field inside the SIW \\
\hline Ref. [19] & $3-14$ & $<-20 \mathrm{~dB}$ & $7.7-19$ & A GSS + HMSIW & Dissipate the fringe EM field of HMSIW \\
\hline Ref. [20] & $3-15$ & $<-15 \mathrm{~dB}$ & $9-40$ & Two GSSs + Microstrip & $\begin{array}{l}\text { Dissipate the fringe EM field on both sides } \\
\text { of microstrip line }\end{array}$ \\
\hline Ref. [21] & $3-15$ & $<-16 \mathrm{~dB}$ & $9-40$ & A GSS + CPW & $\begin{array}{l}\text { Dissipate the EM field around the slot } \\
\text { coupled to the GSS }\end{array}$ \\
\hline Ref. [21] & $3-15$ & $<-15 \mathrm{~dB}$ & $9-40$ & A GSS + slot line & $\begin{array}{l}\text { Dissipate the EM field around the slot } \\
\text { coupled to the GSS }\end{array}$ \\
\hline
\end{tabular}

intrinsic strength [16], high electron mobility [17], etc. Owing to these intrinsic excellent characteristics, meanwhile, the CVD synthesis method guarantees the production of the high quality and large-scale graphene films. Hence, the microwave devices based on CVD-grown graphene have been studied widely and constantly [14].

\section{Attenuators based on CVD-grown graphene}

Due to the possibility to electronically modify the surface impedance of graphene at microwave frequencies, graphene can substitute for non-tunable lossy material, such as resistive film. Hence, most attenuators based on CVDgrown graphene for microwave application have an advantage of the tunable characteristic. For the present, the dynamically tunable attenuators based on CVD-grown graphene have been mainly proposed by the Lu et al. In the research of this group, each proposed attenuator is composed of a kind of transmission line and one or several graphene sandwich structures (GSSs) [18-21]. The GSS is a mutually gated graphene capacitor using two monolayer graphene and an ionic liquid electrolyte sandwiched between them [22]. The conductivity of GSS can be dynamically changed with the biased voltage, which leads to the tunable insertion loss of attenuators. Performance comparison of these tunable attenuators based on CVDgrown graphene is listed in Table 2.

In [18], a tunable graphene-based substrate integrated waveguide (SIW) attenuator (GSIWA) operating from 7 to $14.5 \mathrm{GHz}$ has been proposed. Figure 2a presents the geometry of the GSIWA. In the GSIWA, two GSSs, as two conductivity-tunable E-plane septa to dissipate the EM field inside the SIW, are parallelly inserted in the substrate of the SIW along longitudinal direction. When the biased voltage is changed from 0 to $4.0 \mathrm{~V}$, the GSIWA presents the tunable attenuation from 2 to $15 \mathrm{~dB}$ with a stable $7.5 \mathrm{GHz}$ wideband. Based on this, a half-mode substrate integrated waveguide (HMSIW) attenuator operating at $7.7-19 \mathrm{GHz}$ has been presented [19]. Compared with GSIWA, the fabrication of the HMSIW attenuator was simpler. As exhibited in Figure 2b, this attenuator is composed of two $50 \Omega$ microstrip lines, two taper transitions, an HMSIW and a piece of GSS. The attenuation of HMSIW attenuator can be tuned from 3 to $14 \mathrm{~dB}$ continuously with the changing biased voltage.

Since multi-mode EM fields exist in the SIW structure at the high frequency stage, which lead to the bad flatness of attenuation in high frequency band. To overcome the limitation of the SIW structure, the attenuator based on microstrip line, CPW and slot line, which all have an ultrawideband working frequency band from 9 to $40 \mathrm{GHz}$, have been proposed in [20,21] subsequently. For the microstrip line structure, as shown in Figure 2c, the two GSSs were placed on both sides of the microstrip line along the direction of EM wave propagation. Figure $2 \mathrm{~d}$ and e present the geometry of the CPW attenuator and slot line attenuator, respectively, the GSSs are deposited on the CPW or slot line directly. These three attenuators have the same tunable attenuation range from 3 to $15 \mathrm{~dB}$, and the return loss of all maintain the relatively low level in working frequency band.

\section{Absorbers based on CVD-grown graphene}

The development of transparent or tunable microwave absorbers has been limited until recently by the lack of 


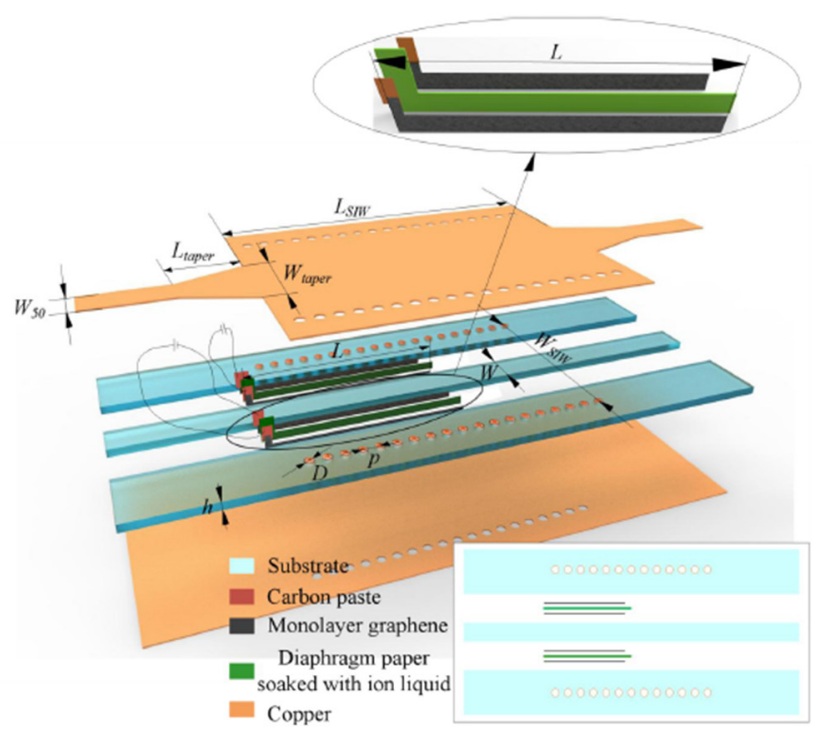

(a)

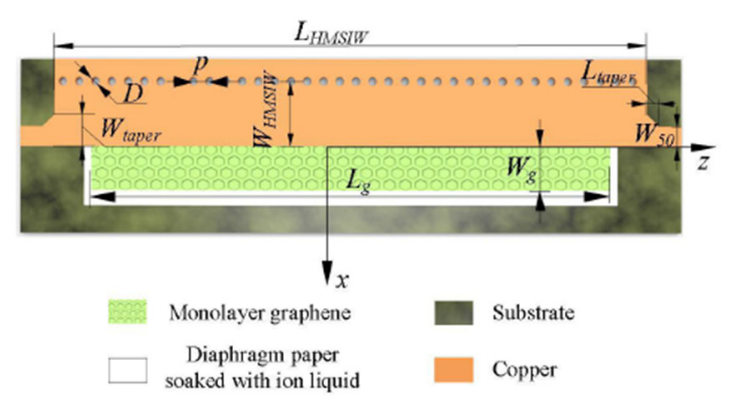

(b)

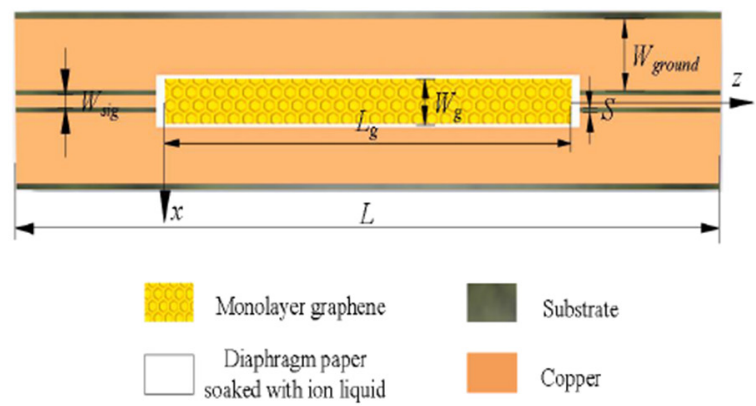

(d)

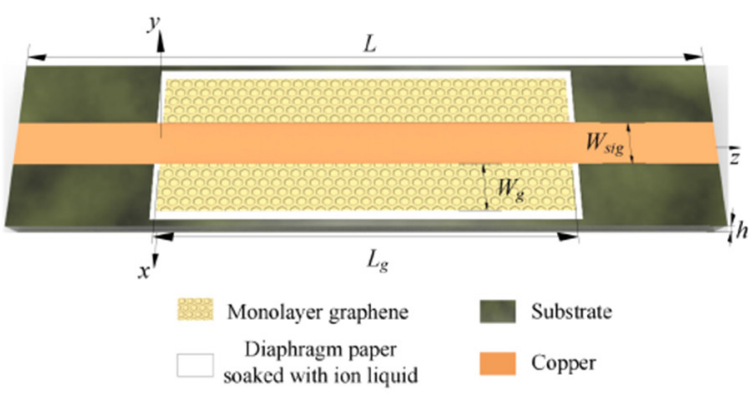

(c)

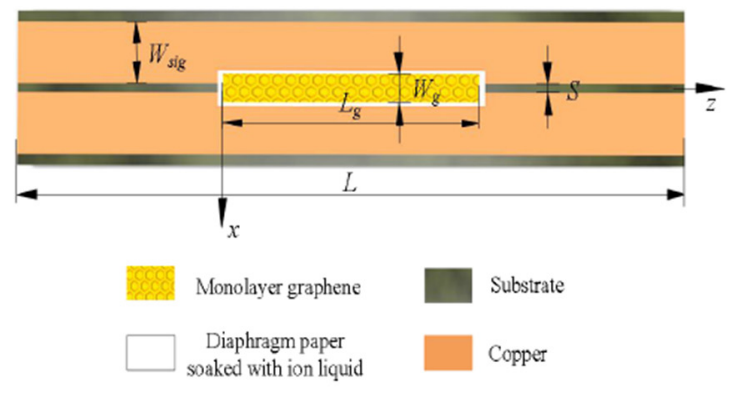

(e)

Fig. 2. (a) The configuration of GSIWA. (b) The configuration of HMSIW attenuator. (c) The configuration of attenuator based on microstrip. (d) The configuration of CPW attenuator. (e) The configuration of slot line attenuator.

suitable materials. Graphene may lead to disruptive innovations in such applications. According to the naturally thin, transparent, frequency-independent and tunable resistance properties of the graphene, it can be a good solution for the absorbing film in transparent, wideband and tunable microwave absorbers. In 2014, $\mathrm{Wu}$ et al. presented a broadband absorber with $28 \%$ relative bandwidth [23]. Figure 3a displays the configura- 


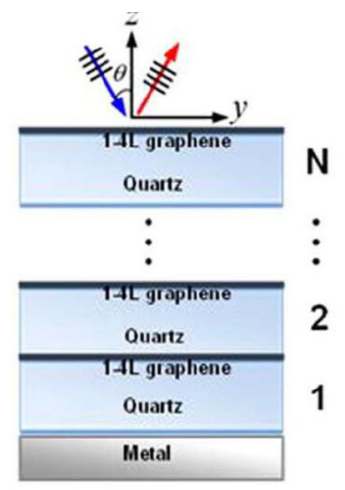

(a)
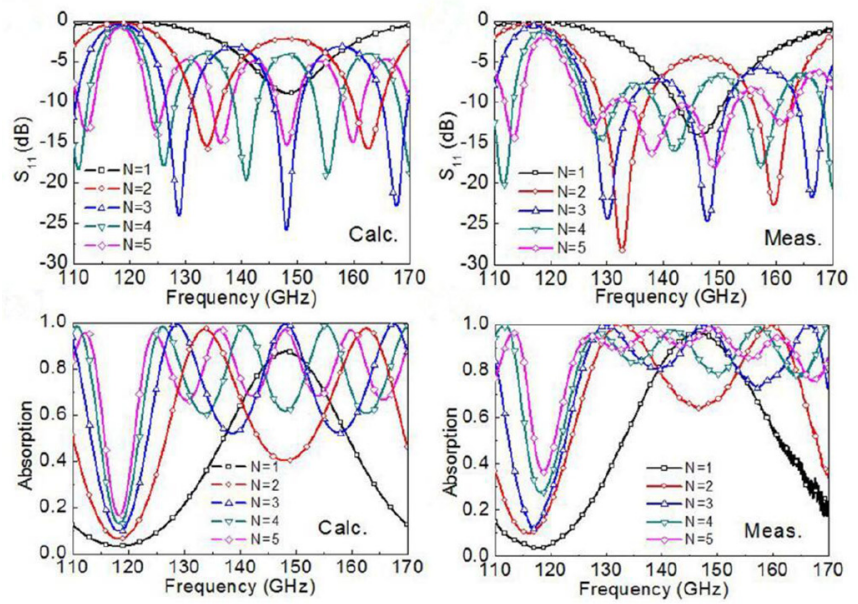

(b)

Fig. 3. (a) The configuration of the broadband absorber. (b) Comparison of calculated and measured $\left|S_{11}\right|$ and absorption of stacked graphene-quartz absorbers.

tion of this absorber. To realize the broadband characteristic of absorber, multilayer graphene-quartz samples are placed on the top of ground plate, which utilizes a series of a quarter wavelength thick dielectric layers that separate parallel resistive sheets to introduce a mutual coupling of Fabry-Perot resonators over a metallic ground. As shown in Figure 3b, for the 5-unit stacked absorber, approximately $90 \%$ absorption can be achieved for $125-165 \mathrm{GHz}$, which indicates the practical millimeter wave absorber has a $28 \%$ fractional absorption bandwidth with the added benefit of optical transparency.

Two years later, four kinds of transparent absorbers with above $80 \%$ optical transmittivity were proposed in $[24,25]$ by Wei et al. The earliest proposed absorber by this group, exhibited in Figure 4a, is composed of monolayer graphene, glass and fluorine-doped tin oxide (FTO) glass as absorbing layer, substrate and reflector layer respectively to form a Salisbury absorber [24]. The measurement results indicate that the absorber can absorb $95 \%$ incident power at $14.5 \mathrm{GHz}$. As shown in Figure 4b, a novel absorber, with the graphene layer patterned with periodic patches to obtain a capacitive and resistive surface, has been presented in [25] subsequently. This absorber possesses the peak absorption coefficient of 0.9 at

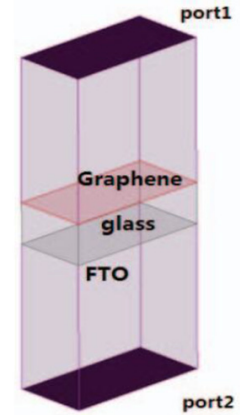

(a)

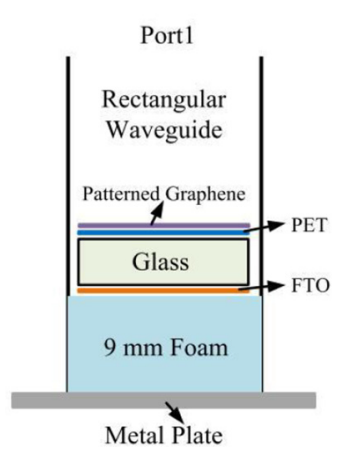

(c)

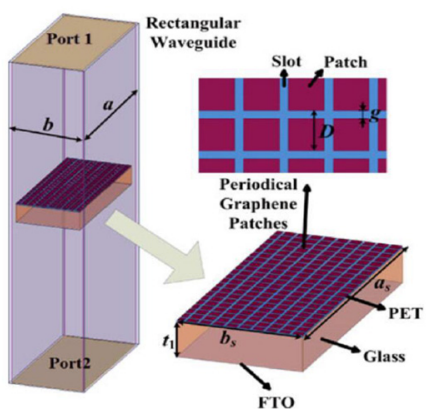

(b)

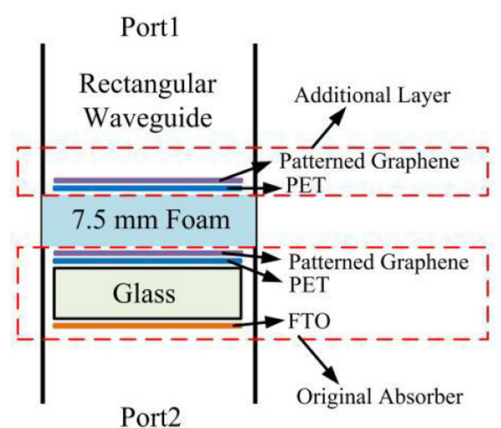

(d)
Fig. 4. (a) The configuration of the absorber based on graphene. (b) The configuration of the absorber based on patterned graphene. (c) The configuration of the absorber based on patterned graphene with metal plate. (d) The configuration of the absorber with double-layer patterned graphene.

12.6 GHz. In order to enhance the absorption, this paper has also displayed a kind of absorber depicted in Figure 4c, which has $100 \%$ absorption coefficient at $12.6 \mathrm{GHz}$. Compared with the structure of previous absorber, in this paper the absorption enhancement of absorber has been realized by placing a metal plate below the FTO layer to reduce the wave penetration. In addition, a broadband absorber is proposed at the end of [25]. The configuration of broadband absorber is shown in Figure 4d, an additional patterned graphene layer is placed at the distance of $7.5 \mathrm{~mm}$ above the original graphene film to change the input impedance of the absorber. Taking the absorption of $80 \%$ as a reference, this absorber has a $3 \mathrm{GHz}$ bandwidth enhancement compared with the first one presented in this paper.

In 2017, the research group of Wei et al. proposed two kinds of absorbers to predict the possibility of tunable absorbers [26]. The center frequency of the absorbers can be changed by the different surface impedance of graphene (TSR approach) exhibited in Figure 5a or the different numbers of graphene layers (SGM approach) exhibited in Figure 5c due to their influence on the real part and imaginary part of the input impedance of the structure. As shown in Figure 5b and d, the measurement results demonstrate that the two absorbers obtain a center frequency shift range of $0.8 \mathrm{GHz}$ for the TSR absorber and $1.2 \mathrm{GHz}$ for the SGM absorber. 


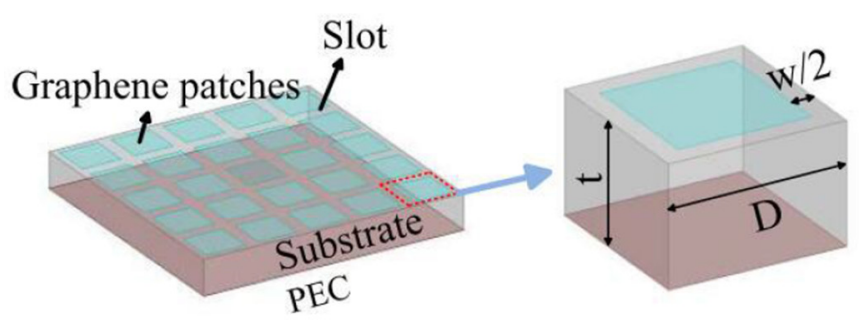

(a)

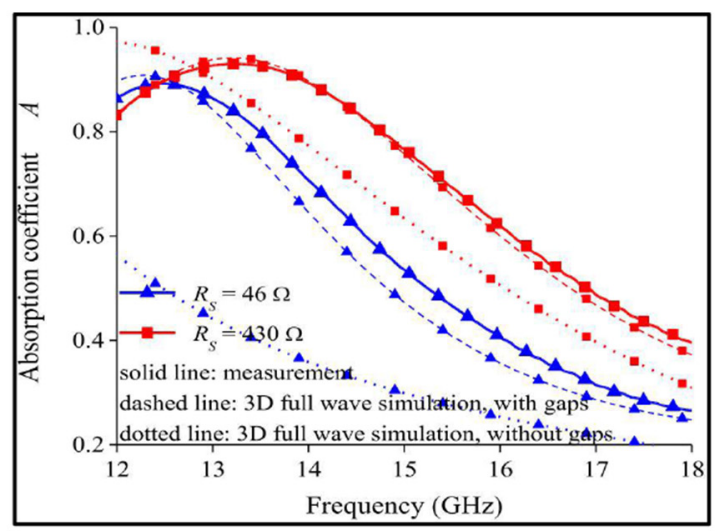

(b)

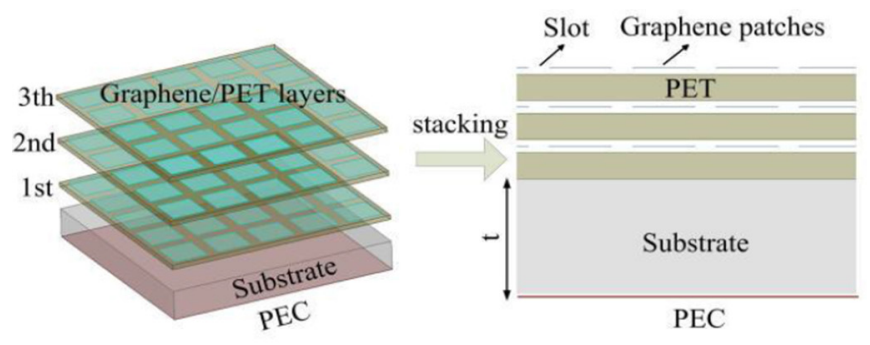

(c)

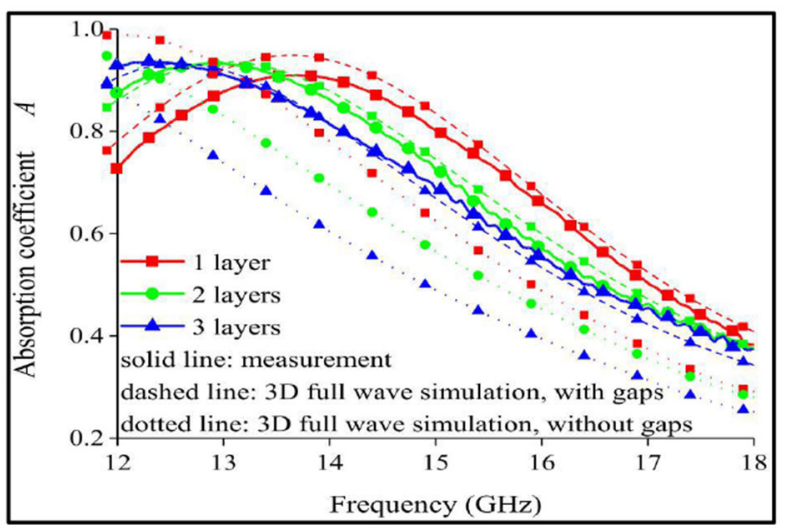

(d)

Fig. 5. (a) The configuration of absorber based on the approach of tuning surface resistance (TSR) and its unit cell. (b) Measured and simulated absorption coefficients of the TSR absorber under the different surface impedance of graphene. (c) The configuration of absorber based on the approach of stacking graphene metasurface (SGM). (d) Measured and simulated absorption coefficients of the SGM absorber when stacking 1, 2 and 3 graphene films.
In 2018, Lu et al. introduced two kinds of absorbers with a planar size of $150 \mathrm{~mm}^{*} 150 \mathrm{~mm}$, which is the first time to experimentally realize such a large-area frequency selective surface using CVD-grown multilayer graphene [27]. Figure $6 \mathrm{a}$ and $\mathrm{b}$ show the absorbers structure and the detail patterns of multilayer graphene which are based on complementary cross-shaped patterns in (Fig. 6a) and Jerusalem cross patterns in (Fig. 6b), respectively. In both structures, the graphene is transferred to polyvinyl chloride (PVC) lay, and then is placed on the top surface of FR4 substrate backed with PEC. Figure 6c and d display the performance of absorbers, the absorption coefficient is changed by different graphene with different surface impedance due to different growth temperature. The absorption coefficient of Type-A increases from below 0.2 to above 0.9 at $13.2 \mathrm{GHz}$. Type-B absorber behaves as a dual-band absorber when multilayer graphene has low surface impedance and as a broadband absorber when multilayer graphene has high surface impedance.

\section{Antennas based on CVD-grown graphene}

The antennas based on CVD-grown graphene for microwave applications have been less exploited because the surface impedance of graphene produced by CVD synthesis method would be generally high, which results in the low radiation efficiency and gain of antenna. However, considering the tunable resistance property of monolayer graphene and the resistance property of multilayer graphene, it could be a candidate for tunable antenna designs, such as the tunable bandwidth, or use for impedance matching of antenna.

In [28], a tunable slot antenna based on CVD-grown graphene operating from 8 to $12 \mathrm{GHz}$ has been proposed by Dragoman et al. As displayed in Figure 7a, the antenna is designed based on coplanar waveguide configuration and fabricated on the 4 inch high resistivity silicon wafer, covered by a $300 \mathrm{~nm} \mathrm{SiO}_{2}$ layer on which the graphene is transferred. In the geometry of antenna, the rectangular patch made from graphene is located in the center position. The signal strip used for exciting the patch and the ground isolated from the patch by the slot are made of the gold material. Figure $7 \mathrm{~b}$ depicts that the $\left|S_{11}\right|$ of antenna is shifted up and down when the surface impedance of graphene is changed by the biased voltage. In contrast with the metal antenna at same configuration and operating frequency, this antenna has wide working band, and has the tunable matching characteristic due to the graphene with the variable surface impedance.

In the same year, the same team proposed another kind of tunable slot antenna based on graphene operating from 9.3 to $10.435 \mathrm{GHz}\left(\left|S_{11}\right|<-10 \mathrm{~dB}\right)$ [29]. The configuration of this antenna is exhibited in Figure 8a. Compared with the antenna proposed in [28], the graphene patch, to change the electric field distribution inside the slot, is placed in the slot instead of in the center of the antenna. The measurement results which is displayed in Figure 8b and c prove the antenna has a tunable range of $\left|S_{11}\right|$ about $20 \mathrm{~dB}$ and a $7.5 \mathrm{MHz}$ shift of the center frequency. 


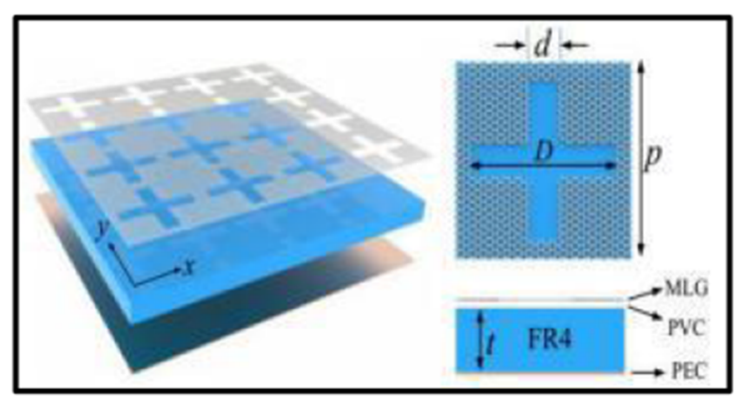

(a)

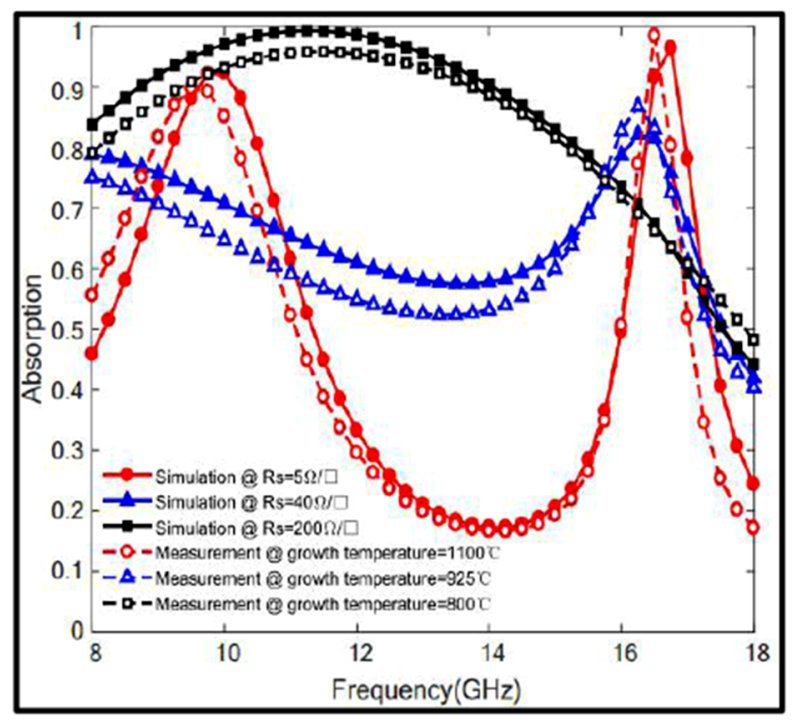

(c)

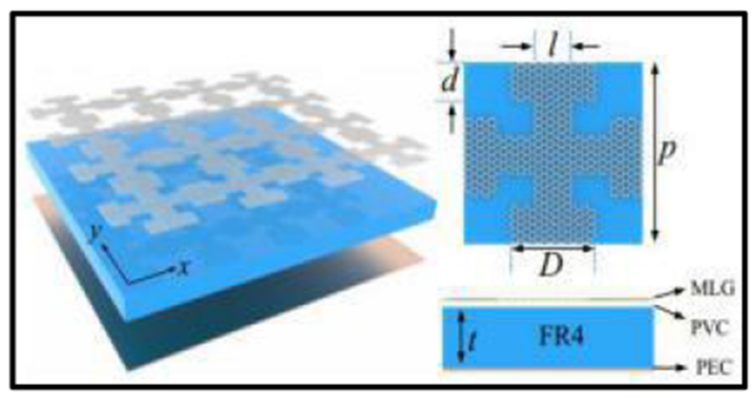

(b)

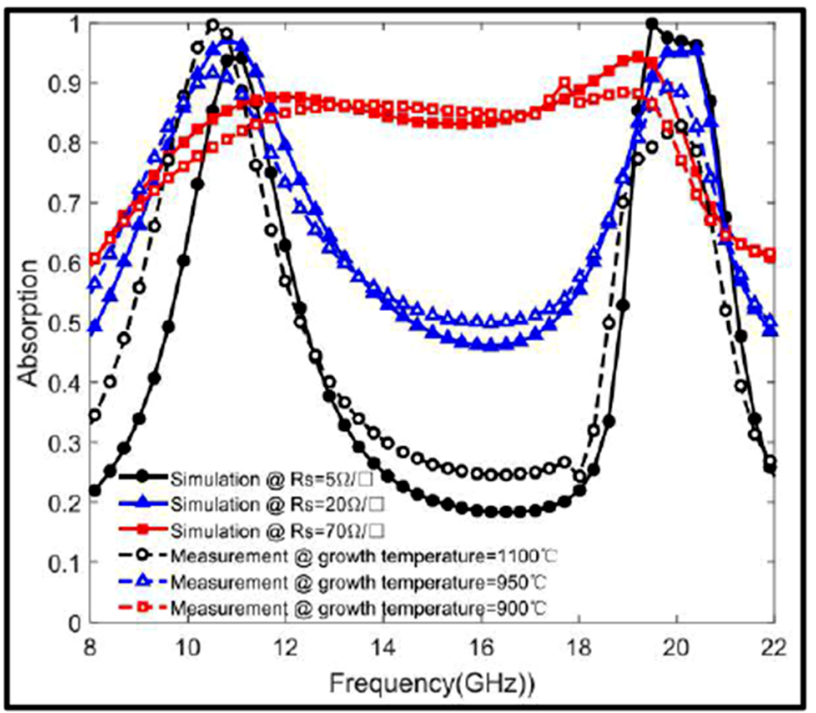

(d)

Fig. 6. (a) The configuration of absorber based on complementary cross-shaped patterns (Type-A). (b) The configuration of absorber based on Jerusalem cross patterns (Type-B). (c) The measured and simulated results of Type-A with different surface impedance of graphene. (d) The measured and simulated results of Type-B with different surface impedance of graphene.

After a period of time, Wei et al. have proposed a dipole antenna loaded the graphene sheets [30]. The geometry of dipole antenna is displayed in Figure 9a. The two graphene sheets as the load are connected with the both ends of the dipole antenna, respectively. The measurement results of this antenna with graphene and without graphene are shown in Figure 9b, indicating that the graphene can improve the impedance matching of the antenna, in which the band of $\left|S_{11}\right|<-10 \mathrm{~dB}$ obtains a $14.9 \%$ increase with graphene.

\section{Other devices based on CVD-grown graphene}

Apart from the application for attenuators, absorbers and antennas, the CVD-grown graphene can also be used in EMI shielding or the beam configuration field. In 2016, Wu et al. proposed a transparent electromagnetic shielding enclosure based on CVD-grown graphene [31]. It can be observed from Figure 10 that the monolayer graphene with the surface impedance of $500 \Omega / \square$ on the quartz substrate is attached to an enclosure made of indium tin oxide (ITO). Through the experimental validation of the transmission coefficients $\left(S_{21}\right)$ between the two ports, as shown in Figure $10 \mathrm{~b}$, the transmission peak of $\mathrm{TE}_{101}$ and $\mathrm{TE}_{201}$ mode are suppressed by about $10 \mathrm{~dB}$ more and $5 \mathrm{~dB}$ more respectively while the shielding enclosure maintains good transparency to visible light.

Recently, Lu et al. have presented a novel graphene ribbon structure to realize the beam reconfiguration [32]. Compared to conventional metallic materials, the biggest advantage of graphene is the variable impedance controlled by different doping or growth environment, this novel property really provides a new method to acquire different phase responses with fixed structure. Figure 11a and b exhibit the unit cell of the graphene ribbons and the configuration of the graphene ribbon array. As the paper described, when the surface impedance of graphene in the unit cell is changed from 5 to $2000 \Omega / \square$, the reflection response of unit cell can achieve a phase difference of $\pi$ and a uniform amplitude at $13 \mathrm{GHz}$. Based on this feature, 


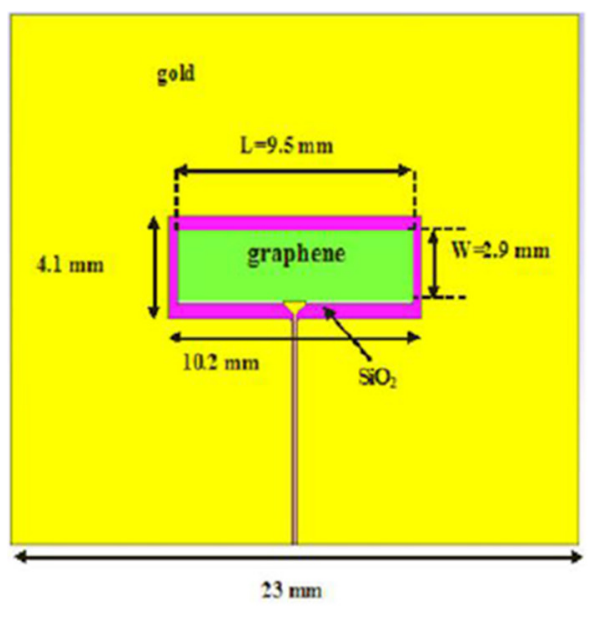

(a)

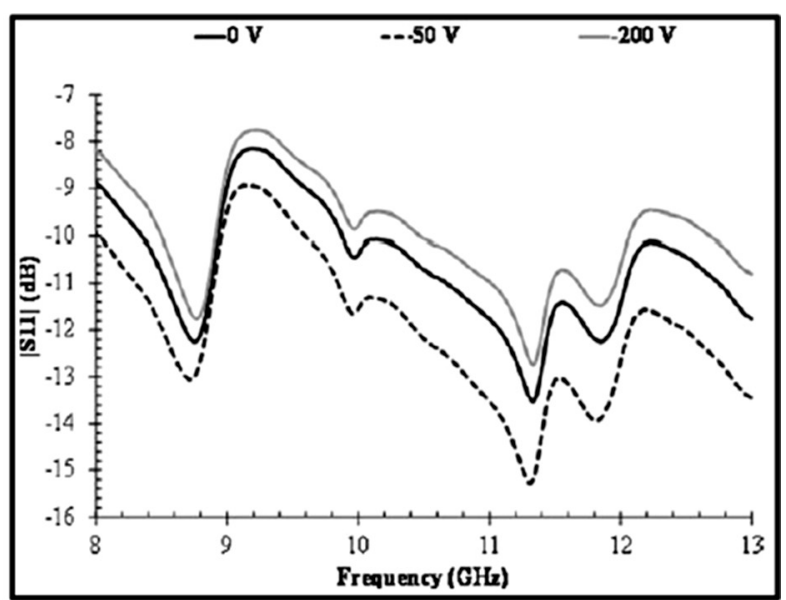

(b)

Fig. 7. (a) The configuration of the tunable microwave slot antenna. (b) Measured $\left|S_{11}\right|$ of antenna in X band with three different bias voltages.

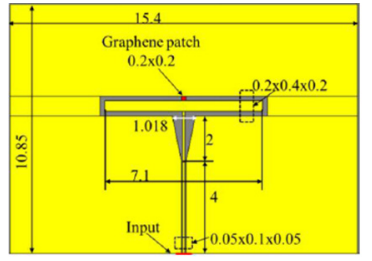

(a)

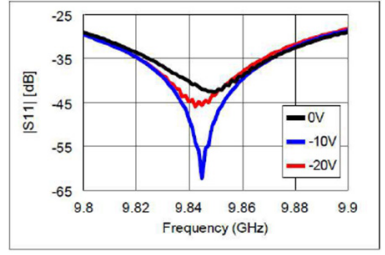

(b)

\begin{tabular}{|c|c|c|}
\hline $\begin{array}{c}\text { Resonance } \\
\text { frequency }[\mathrm{GHz}]\end{array}$ & $|\mathbf{S 1 1}|[\mathbf{d B}]$ & Applied voltage \\
\hline $9.85 \mathrm{GHz}$ & $-42.78 \mathrm{~dB}$ & $0 \mathrm{~V}$ \\
\hline $9.845 \mathrm{GHz}$ & $-62.3 \mathrm{~dB}$ & $-10 \mathrm{~V}$ \\
\hline $9.8425 \mathrm{GHz}$ & $-46.05 \mathrm{~dB}$ & $-20 \mathrm{~V}$ \\
\hline
\end{tabular}

(c)

Fig. 8. (a) The configuration of the $\mathrm{X}$ band slot antenna. (b) Measured $\left|S_{11}\right|$ parameters with different bias voltages. (c) The detailed $\left|S_{11}\right|$ parameters with different bias voltages of the center frequency points.

different angles of reflected lobes could be realized by properly arranging the graphene ribbon arrays by experimental demonstration, which indicates that the graphene can be a good choice for beam reconfiguration.

\section{Conclusion}

In this paper, we have reviewed the special graphene structure, the four kinds of synthesis methods and the excellent properties in the beginning. Due to the naturally thin, transparent, frequency-independent and tunable resistance properties of the graphene, meanwhile, the CVD synthesis method guarantees the production of the high quality and large-scale graphene films, thus microwave devices based on graphene produced by CVD-grown
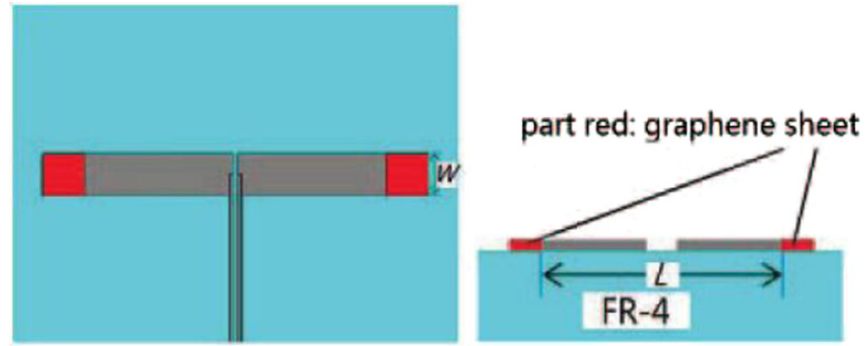

(a)

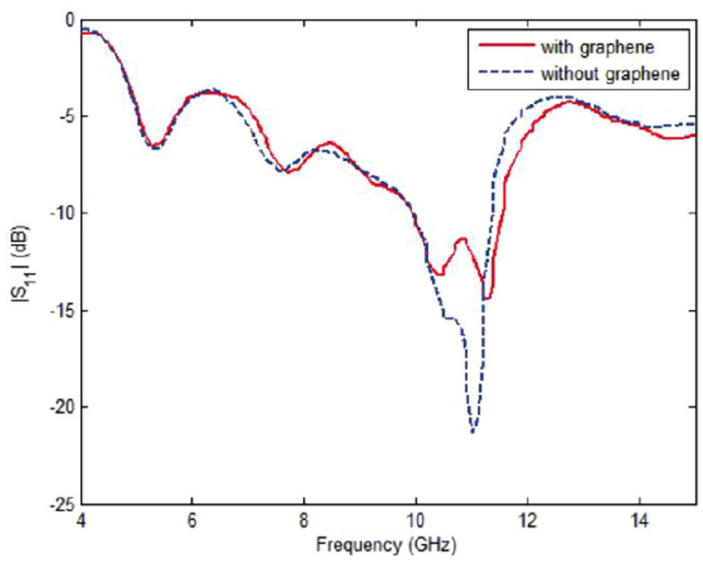

(b)

Fig. 9. (a) The configuration of the dipole antenna. Red part is the graphene sheets. (b) Measured $\left|S_{11}\right|$ of the dipole antenna with graphene and without antenna.

synthesis method have been studied widely and constantly. This paper has presented an overview of the graphene based on CVD method applied to attenuators, absorbers, antennas, electromagnetic shielding enclosure and beam 


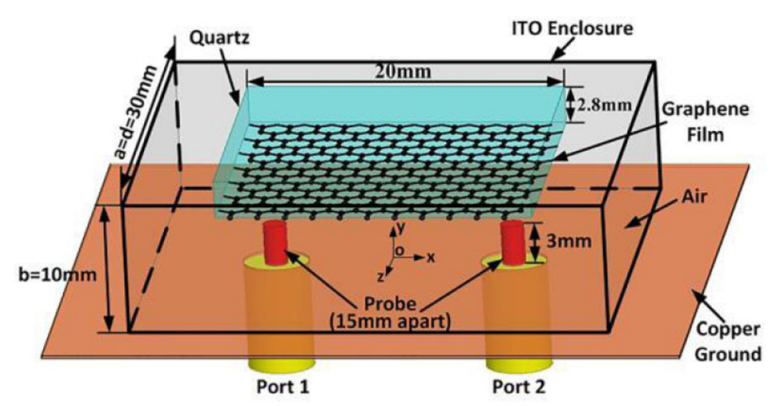

(a)

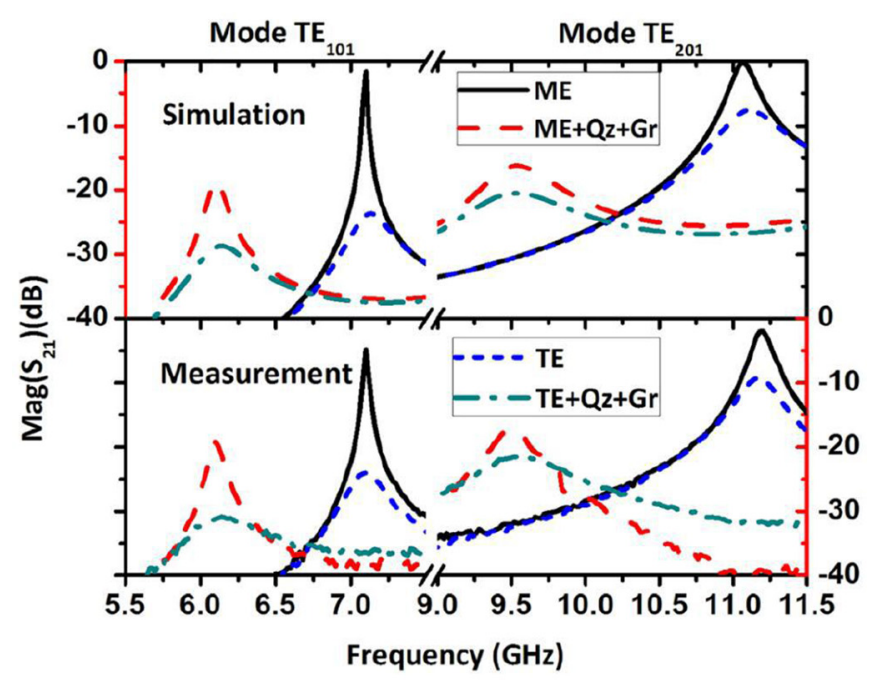

(b)

Fig. 10. (a) The configuration of the transparent electromagnetic shielding enclosure. (b) Simulated and measured transmission coefficients of the metallic enclosure (ME) and ITO transparent enclosure (TE). The status of the two enclosures after graphene (Gr) film and quartz (Qz) imbedded is illustrated.

reconfiguration, which are mainly researched by Chinese scientific groups. From these researches, we can find the huge scientific research potential of this emerging material.

\section{References}

1. A.K. Geim, K.S. Novoselov, The rise of graphene, Nat. Mater. 6, 183 (2007)

2. S. Edwards Rebecca, S. Coleman Karl, Graphene synthesis: relationship to applications, Nanoscale 5, 38 (2013)

3. A.H. Castro Neto, F. Guinea, N.M.R. Peres et al., The electronic properties of graphene, Rev. Mod. Phys. 81, 109 (2009)

4. K.S. Novoselov, V.I. Fal'Ko, L. Colombo, P.R. Gellert, M.G. Schwab, K. Kim, A roadmap for graphene, Nature 490, 192 (2012)

5. K.S. Novoselov, A.K. Geim, S.V. Morozov, D. Jiang, V. Zhang, S.V. Dubonos, I.V. Grigorieva, A.A. Firsov, Electric field effect in atomically thin carbon films, Science 306, 666 (2004) (a)

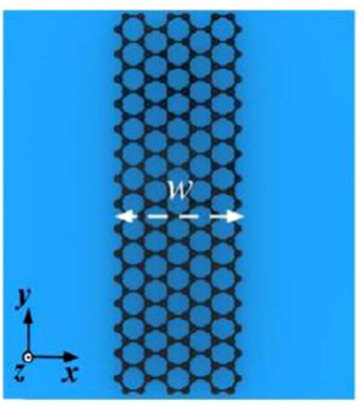

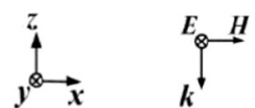

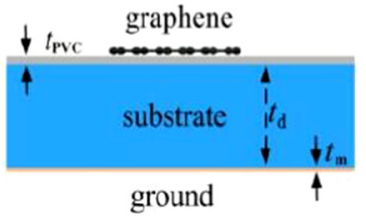

(b) (a)

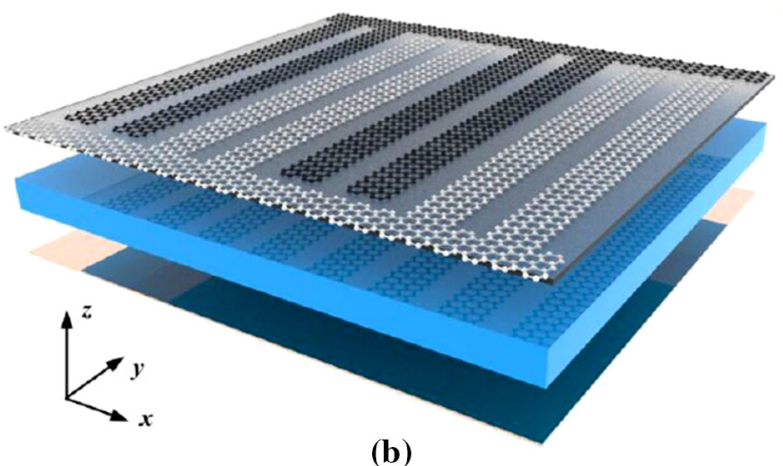

(b)
Fig. 11. (a) The configuration of the unit cell of the graphene ribbons. (b) The configuration of the graphene ribbon array.

6. J. Kedzierski, P.-L. Hsu, P. Healey et al., Epitaxial graphene transistors on SIC substrates, IEEE Trans. Electron Devices 55, 2078 (2008)

7. S. Stankovich, D.A. Dikin, H.B. Dommett Geoffrey et al., Graphene-based composite materials, Nature 442, 282 (2006)

8. S. Stankovich, D.A. Dikin, R.D. Piner et al., Synthesis of graphene-based nanosheets via chemical reduction of exfoliated graphite oxide, Carbon 45, 1558 (2007)

9. M. Dragoman, D. Neculoium, D. Dragoman, G. Deligeorgis, G. Konstantinidis, A. Cismaru, F. Coccetti, R. Plana, Graphene for microwave, IEEE Microw. Mag. 11, 81 (2010)

10. V.C. Tung, M.J. Allen, Y. Yang, R.C. Kaner, Highthroughput solution processing of large-scale graphene, Nat. Nanotechnol. 4, 2 (2009)

11. S. Bae, H. Kim, Y. Lee, X. Xu, J.-S. Park, Y. Zheng, Roll-toroll production of 30-inch graphene films for transparent electrodes, Nat. Nanotechnol. 5, 574 (2010)

12. M. Bozzi, L. Pierantoni, S. Bellucci, Applications of Graphene at microwave frequencies, Radioengineering 24, 661 (2015)

13. A.K. Geim, Graphene: status and prospects, Science 324, 1530 (2009)

14. L. Pierantoni, D. Mencarelli, M. Bozzi, R. Moro, S. Bellucci, Microwave applications of graphene for tunable devices, in: 44th European Microwave Conference, Rome, Italy, 6-9 October 2014, pp. 1456-1459

15. A.A. Balandin, Thermal properties of graphene and nanostructured carbon materials, Nat. Mater. 10, 569 (2011)

16. C. Lee, X. Wei, J.W. Kysar, J. Hone, Measurement of the elastic properties and intrinsic strength of monolayer graphene, Science 321, 385 (2008)

17. K.I. Bolotin et al., Ultrahigh electron mobility in suspended graphene, Solid State Commun. 146, 351 (2008) 
18. A.Q. Zhang, W.B. Lu, Z.G. Liu, H. Chen, B.H. Huang, Dynamically tunable substrate-integrated-waveguide attenuator using graphene, IEEE Trans. Microw. Theory Tech. 66, $3081(2018)$

19. A.Q. Zhang, Z.G. Liu, W.B. Lu, H. Chen, Graphene-based dynamically tunable attenuator on a half-mode substrate integrated waveguide, Appl. Phys. Lett. 112, 161903 (2018)

20. A.Q. Zhang, Z.G. Liu, W.B. Lu, H. Chen, Dynamically tunable attenuator on graphene-based microstrip line, IEEE Trans. Microw. Theory Tech. (2018). DOI: 10.1109/ TMTT.2018.2885761 (early access)

21. A.Q. Zhang, Z.G. Liu, H. Chen, W.B. Lu, Graphene-based dynamically tunable attenuator on a coplanar waveguide or a slot line, IEEE Trans. Microw. Theory Tech. 67, 70 (2019)

22. O. Balci, E.O. Polat, N. Kakenov, C. Kocabas, Graphene enabled electrically switchable radar-absorbing surfaces, Nat. Commun. 6, 1 (2015)

23. B. Wu, H.M. Tuncer, M. Naeem, B. Yang, M.T. Cole, W.I. Milne, Y. Hao, Experimental demonstration of a transparent graphene millimeter wave absorber with $28 \%$ fractional bandwidth at $140 \mathrm{GHz}$, Sci. Rep. 4, 4130 (2014)

24. D. Yi, X. Wei, Y. Xu, Experimental demonstration of transparent microwave absorber based on graphene, in: IEEE MTT-S International Wireless Symposium Digest, Shanghai, China, 2016, pp. 1-2
25. D. Yi, X.C. Wei, Y.L. Xu, Transparent microwave absorber based on patterned graphene: design, measurement, and enhancement, IEEE Trans. Nanotechnol. 16, 484 (2017)

26. D. Yi, X.-C. Wei, Y.-L. Xu, Tunable microwave absorber based on patterned graphene, IEEE Trans. Microw. Theory Tech. 65, 2819 (2017)

27. H. Chen, W.B. Lu, Z.G. Liu, J. Zhang, A.Q. Zhang, B. Wu, Experimental demonstration of microwave absorber using large-area multilayer graphene-based frequency selective surface, IEEE Trans. Microw. Theory Tech. 66, 3807 (2018)

28. M. Dragoman et al., A tunable microwave slot antenna based on graphene, Appl. Phys. Lett. 106, 153101-1 (2015)

29. A.-C. Bunea, D. Neculoiu, G. Konstantinidis, G. Deligiorgis, $\mathrm{X}$-band tunable slot antenna with graphene patch, in: Proceedings of 2015 European Microwave Conference, 2015, pp. $614-617$

30. D. Li, X.C. Wei, N. Meng et al., Exploring graphene loaded antenna for $\mathrm{GHz}$ potential applications by experiment, in: 2015 Asia Pacific Microwave Conference (APMC), Nanjing, China, 6-9 December 2015

31. Y.T. Zhao, B. Wu, Y. Zhang, Y. Hao, Transparent electromagnetic shielding enclosure with CVD grapheme, Appl. Phys. Lett. 109, 1 (2016)

32. H. Chen, Z.G. Liu, W.B. Lu, A.Q. Zhang, X.B. Li, Microwave beam reconfiguration based on graphene ribbon, IEEE Trans. Antennas Propag. 66, 6049 (2018)

Cite this article as: Wei-Bing Lu, Hui Chen, Zhen-Guo Liu, A review of microwave devices based on CVD-grown graphene with experimental demonstration, EPJ Appl. Metamat. 6, 8 (2019) 